\title{
Collective modes of an imbalanced trapped Fermi gas in two dimensions at finite temperatures
}

\author{
S. N. Klimin, ${ }^{1, *}$ J. Tempere, ${ }^{1,2}$ J. T. Devreese, ${ }^{1, \dagger}$ and B. Van Schaeybroeck ${ }^{3,4}$ \\ ${ }^{1}$ Theorie van Kwantumsystemen en Complexe Systemen (TQC), Universiteit Antwerpen, Universiteitsplein 1, B-2610 Antwerpen, Belgium \\ ${ }^{2}$ Lyman Laboratory of Physics, Harvard University, Cambridge, Massachusetts 02138, USA \\ ${ }^{3}$ Koninklijk Metereologisch Instituut (KMI), Ringlaan 3, B-1180 Brussels, Belgium \\ ${ }^{4}$ Instituut voor Theoretische Fysica, Katholieke Universiteit Leuven, Celestijnenlaan 200 D, B-3001 Leuven, Belgium
}

(Received 28 January 2011; revised manuscript received 18 May 2011; published 30 June 2011)

\begin{abstract}
Phase diagrams and collective excitations are investigated for a trapped imbalanced Fermi gas in two dimensions with $s$-wave pairing at finite temperatures. The treatment of collective modes is performed within the hydrodynamic approach using the Euler and continuity equations. The equations of state for different phases are simulated by a polytropic law with parameters obtained using a fit to the microscopic equilibrium distributions of the pressure and density. These are determined within the whole range of the BCS-BEC crossover using a path-integral description and taking into account fluctuations about the saddle point. We focus on the case of imbalanced gases, when the number of "spin-up" and "spin-down" fermions that form the pair is not equal. The superfluid-to-normal transition in the trapped Fermi gas is governed by the Berezinskii-Kosterlitz-Thouless mechanism. The eigenfrequencies of collective modes behave differently from those in the zero-temperature case. They can be used to study a realistic equation of state of a trapped Fermi gas at finite temperatures.
\end{abstract}

DOI: 10.1103/PhysRevA.83.063636

PACS number(s): 03.75.Ss, 05.30.Fk, 03.75.Lm

\section{INTRODUCTION}

Recent experiments on pairing in ultracold Fermi gases resulted in a successful realization of superfluid states in different quasidimensionalities. Substantial attention has been paid to the experimental study of cold boson and fermion atoms confined to optical lattices, which allow us to control the confinement geometry and dimensionality. The experimental realization of superfluidity in Bose gases in optical lattices $[1,2]$ has been followed by observations of superfluid states of low-dimensional Fermi gases [3].

The superfluid regime is reached in two-dimensional (2D) systems through the Berezinskii-Kosterlitz-Thouless (BKT) phase transition [4-6]. The BKT transition has been recently observed by Hadzibabic et al. [7,8] in a trapped quantum degenerate gas of ${ }^{87} \mathrm{Rb}$. This has stimulated renewed theoretical interest in the BKT transition in trapped dilute gases (for a review, see Ref. [9]). The phase diagram for balanced 2D and quasi-2D Fermi gases with $s$-wave pairing has been theoretically investigated, e.g., in Refs. [10-12]. In Refs. [13,14], the approach of Refs. [10-12] has been extended to the case of a two-component Fermi gas with unequal "spin-up" and "spin-down" populations.

In Refs. [10,11,13], the fermion pairing is considered, taking into account phase fluctuations and neglecting ad hoc amplitude fluctuations. This is based on the assumption that to describe the BKT transition, one can in a first approximation focus on phase fluctuations only [9], which is well-founded at sufficiently low temperatures. In Ref. [14], the BKT phase transition in a 2D imbalanced fermion system has been studied taking into account both amplitude and phase fluctuations, and

\footnotetext{
* On leave from Department of Theoretical Physics, State University of Moldova, str. A. Mateevici 60, MD-2009 Kishinev, Republic of Moldova.

${ }^{\dagger}$ Also at Technische Universiteit Eindhoven, P. O. Box 513, 5600 MB Eindhoven, The Netherlands.
}

the effect of amplitude fluctuations at finite temperatures was quantified.

The investigation of collective oscillations in cold gases offers insights into the properties of strongly correlated systems. Experiments on collective modes in ultracold trapped Fermi gases [15-21] reveal the different regimes that can be realized by controlling both the temperature of the gas and the interaction strength between the atoms by means of a Feshbach resonance.

In Refs. [22,23], collective excitations for trapped imbalanced $3 \mathrm{D}$ and $2 \mathrm{D}$ fermion gases at zero temperature have been investigated within the mean-field theory for homogeneous phases. In the present work, we treat collective excitations for trapped imbalanced Fermi gases in 2D at finite temperatures. Collective excitations in trapped Fermi gases with a population imbalance are especially interesting for experiment, because a variation of the population imbalance at finite nonzero temperatures allows experimentalists to probe different superfluid phases and measure the equation of state in a wide range of densities.

There are many theoretical investigations of collective excitations in cold Fermi gases based on the hydrodynamic approximation [24-32]. In these works, a polytropic approximation for the equation of state is used since it can provide analytical results for eigenfrequencies of collective modes. The exponents and prefactors used in the polytropic equation of state for the superfluid and the normal fluids are commonly chosen equal to limiting case values. Here, we use a microscopic description to obtain results for the equation of state, and fit polytropic laws to it to extract the polytropic parameters in the whole region of interaction strengths and imbalance. The microscopic distributions are determined here within the path-integral formalism of Refs. $[13,14]$ within the whole range of the BCS-BEC crossover taking into account Gaussian fluctuations about the saddle point. This formalism is suited for both $T=0$ and nonzero temperature. In phase diagrams for the homogeneous equilibrium Fermi gas [14], the superfluid phase transition is of the BKT type. In addition 
to the superfluid and normal phases, there is a noncoherent quasicondensate in those phase diagrams. In a trapped Fermi gas, the aforementioned phases are spatially separated from each other. This results in a rich spectrum of collective excitations due to the presence of interfaces between different phases.

The paper is organized as follows. In Sec. II, we consider the path-integral method and phase diagrams for the equilibrium state of interacting imbalanced fermions in 2D with $s$-wave pairing. In Sec. III, the equilibrium configuration of the trapped imbalanced Fermi gas are discussed. In Sec. IV, we present the formalism, results, and discussion for collective excitations. The treatment is followed by conclusions, Sec. V.

\section{MICROSCOPIC PATH-INTEGRAL DESCRIPTION}

\section{A. Theory}

First we consider equilibrium properties of a twocomponent gas of interacting fermions in 2D. Within the path-integral formalism, the partition function is expressed as a path integral over Grassmann variables $\left[\bar{\psi}_{\mathbf{x}, \tau, \sigma}, \psi_{\mathbf{x}, \tau, \sigma}\right]$,

$$
\mathcal{Z} \propto \int \mathcal{D}\left[\bar{\psi}_{\mathbf{x}, \sigma}(\tau), \psi_{\mathbf{x}, \sigma}(\tau)\right] \exp (-S),
$$

where $S$ is the action functional of interacting fermions with $s$-wave pairing and with a contact interaction. The details of the derivation can be found in Ref. [14]. We apply the Hubbard-Stratonovich transformation to the path integral in (1). After integrating out the fermion Grassmann variables, an effective action in Bose fields is obtained. The remaining functional integral over those Bose fields cannot be taken in general. To proceed further, different levels of approximation can be made. The crudest approximation is the mean-field approximation. To improve on this, fluctuations around the saddle point are taken into account. This can be done in an exact way only up to quadratic order in the fluctuations. In Ref. [13], we focused on phase fluctuations in order to treat the quasicondensate state, relevant for the $2 \mathrm{D}$ case. The calculation scheme of Ref. [14] differs from both the mean-field and the hydrodynamic approaches used in Refs. [10,11,13] in that amplitude as well as phase fluctuations are taken into account, analogously to the treatment in three dimensions developed by Nozières and Schmitt-Rink (NSR) [33] (see further developments in Refs. [34-38]).

There are common essential theoretical ingredients between the present approach for the fermion gas in two dimensions and the method of Refs. [34-36] for the same system in 3D. Similarly to those works, the present method is based on the expansion of the effective Hubbard-Stratonovich bosonic action about the saddle point. The zeroth-order term of the expansion is the saddle-point solution, which gives us the mean-field gap equation and number equations. The next-order term of the expansion represents the Gaussian quadratic fluctuations. They are taken into account in the number equations. As shown in Ref. [39], a straightforward extension of the scheme of Refs. [34-36] to the 2D case leads to a long-wavelength divergence of the fluctuation contribution to the fermion density and therefore to the zero critical temperature for the superfluid phase transition. This divergence is related to the Hohenberg-Mermin-Wagner theorem $[40,41]$ on the impossibility of the long-range order in a uniform 2D system. However, a quasi-long-range order can be realized in two dimensions as a quasicondensate [42]. In Refs. [10-12], the fluctuations are taken into account after the gauge transformation of the fermion field $\psi$ and the pair field $\phi$,

$$
\begin{gathered}
\phi(\mathbf{r}, \tau)=\Delta(\mathbf{r}, \tau) e^{i \theta(\mathbf{r}, \tau)}, \\
\psi(\mathbf{r}, \tau)=\chi(\mathbf{r}, \tau) e^{i \theta(\mathbf{r}, \tau) / 2},
\end{gathered}
$$

[where $\Delta(\mathbf{r}, \tau)$ and $\theta(\mathbf{r}, \tau)$ are, respectively, the amplitude and the phase of the pair field] and the subsequent long-wavelength approximation for the phase-fluctuation action. This scheme has been extended to the imbalanced case in Ref. [13]. Further, in Ref. [14] this approach has been updated to include both phase and amplitude fluctuations. The number equations based on the pair fluctuation actions of Refs. [10-14] give a finite fermion density in a paired state. The paired state above the BKT transition temperature is interpreted in Refs. [13,14] as a noncoherent quasicondensate, in which the amplitude of the gap parameter is other than zero while the phase coherence is absent.

The results below are expressed in terms of the averaged chemical potential $\mu=\left(\mu_{\uparrow}+\mu_{\downarrow}\right) / 2$ determining the total number of fermions and the chemical potential imbalance $\zeta=\left(\mu_{\uparrow}-\mu_{\downarrow}\right) / 2$, determining the population imbalance. We use the units in which $\hbar=1$, the fermion mass $m=1 / 2$. As the energy unit, we use the Fermi energy $E_{F}$ of a balanced, noninteracting Fermi gas with the same total density.

For the treatment both with and without fluctuations, the saddle-point thermodynamic potential $\Omega_{\mathrm{sp}}$ is necessary to determine the thermodynamic equilibrium value of the gap. For $s$-wave pairing in 2D with imbalance, the saddle-point thermodynamic potential is given by [43]

$$
\begin{aligned}
\Omega_{\mathrm{sp}}= & -\int \frac{d^{2} \mathbf{k}}{(2 \pi)^{2}}\left\{\frac{1}{\beta} \ln \left[2 \cosh (\beta \zeta)+2 \cosh \left(\beta E_{\mathbf{k}}\right)\right]-\xi_{\mathbf{k}}\right\} \\
& -\frac{1}{g} \Delta^{2}
\end{aligned}
$$

where $g$ is the coupling constant of the fermion-fermion contact interaction, $\xi_{\mathbf{k}}=k^{2}-\mu$ is the fermion energy, and $E_{\mathbf{k}}=\sqrt{\xi_{\mathbf{k}}^{2}+\Delta^{2}}$ is the Bogoliubov excitation energy. The renormalization of the coupling constant is performed following Refs. [43,44]. In two dimensions, as indicated in [44], the strength of the pseudopotential is energy-dependent, $g=g(E)$. The coupling constant is renormalized as

$\frac{1}{g(E)}=\frac{1}{8 \pi}\left(\ln \frac{E_{b}}{E}+i \pi\right)-\int \frac{d^{2} \mathbf{k}}{(2 \pi)^{2}} \frac{1}{2 k^{2}-E+i \delta}$,

where $E_{b}$ is the energy of the $2 \mathrm{D}$ bound state.

The gap parameter $\Delta$ is determined from the gap equation generalized to the imbalance case - the minimum condition for the saddle-point thermodynamic potential as a function of the gap parameter $\Delta$ at fixed temperature and chemical potentials:

$$
\frac{\partial \Omega_{\mathrm{sp}}(\beta, \mu, \zeta, \Delta)}{\partial \Delta}=0 .
$$

For given fermion density $n$ [in the units chosen above, $n=1 /(2 \pi)]$ and relative local density imbalance $\frac{\delta n}{n} \equiv \delta$, the 


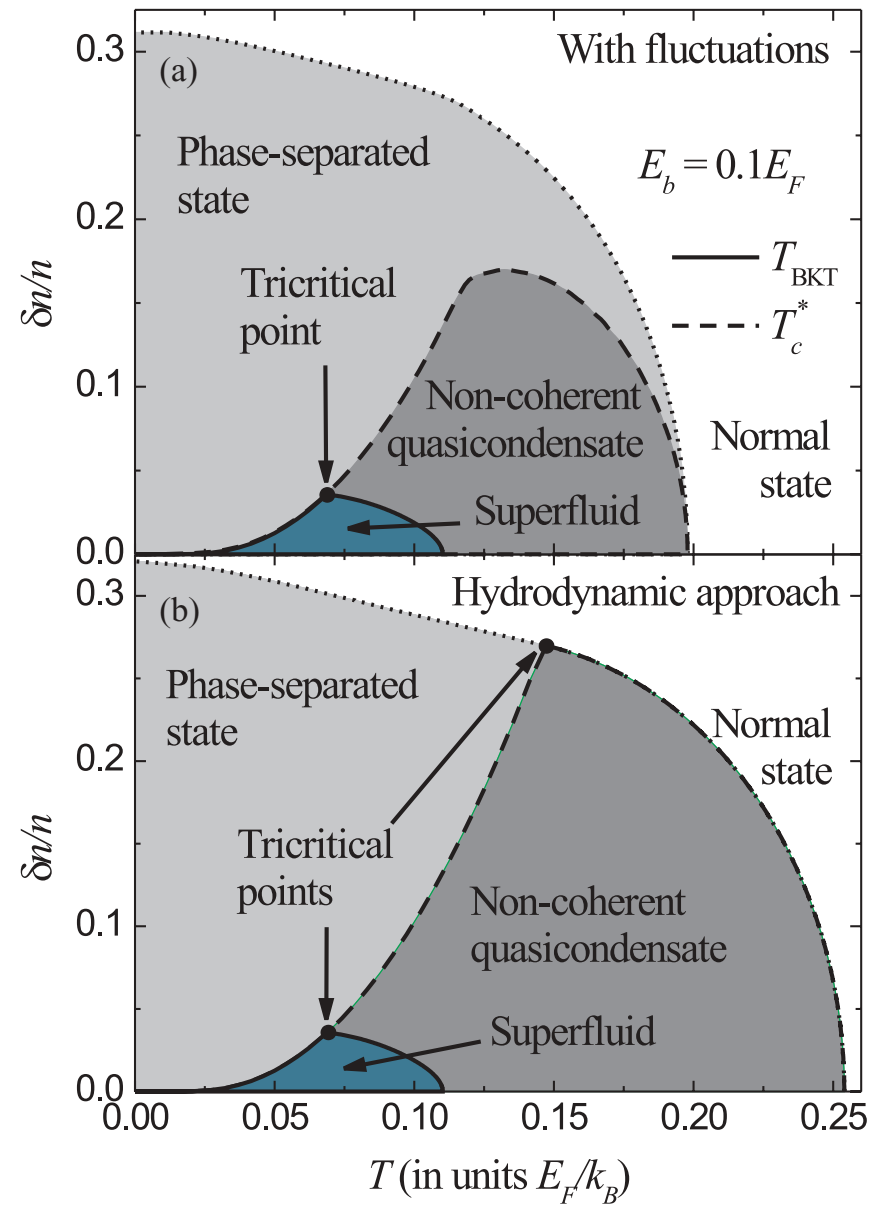

FIG. 1. (Color online) Phase diagrams for a 2D imbalanced Fermi gas at binding energy $E_{b}=0.1 E_{F}$ calculated taking into account both amplitude and phase fluctuations (a) and calculated within the hydrodynamic approximation of Ref. [13] (b). (Figure reprinted from Ref. [14].)

chemical potentials $\mu$ and $\zeta$ are determined from the number equations

$$
\begin{gathered}
n_{\text {sp }}+n_{\text {fluct }}=\frac{1}{2 \pi}, \\
\delta n_{\text {sp }}+\delta n_{\text {fluct }}=\frac{1}{2 \pi} \delta .
\end{gathered}
$$

The number equations (6) and (7) and the generalized gap equation (5) are solved jointly with the equation for the BKT transition temperature $T_{\mathrm{BKT}}$. It is determined from the universal relation [6] as in Ref. [13],

$$
T_{\mathrm{BKT}}-\frac{\pi}{2} \rho_{s}\left(T_{\mathrm{BKT}}\right)=0,
$$

where $\rho_{s}$ is the superfluid pair density. The expression for $\rho_{s}$ (from Ref. [13]) is the extension of the pair superfluid density from Ref. [10] to the case of imbalanced fermion populations:

$$
\begin{aligned}
\rho_{s}= & \frac{1}{2(2 \pi)^{2}} \int d^{2} \mathbf{k}\left[1-\frac{\xi_{k}}{E_{k}} \frac{\sinh \beta E_{k}}{\cosh \beta \zeta+\cosh \beta E_{k}}\right. \\
& \left.-k^{2} \beta \frac{\cosh \beta \zeta \cosh \beta E_{k}+1}{\left(\cosh \beta \zeta+\cosh \beta E_{k}\right)^{2}}\right] .
\end{aligned}
$$

The saddle-point (sp) and fluctuation (fluct) contributions to the fermion density and to the density difference are calculated through the derivatives of the corresponding contributions to the thermodynamic potential density. The explicit expressions for the fermion density and density difference are given in Appendix A.

\section{B. Phase diagrams}

In the present treatment, both amplitude and phase fluctuations are taken into account through the formalism outlined in Ref. [14]. The Kosterlitz-Nelson equation (8) for the BKT temperature remains unchanged by the inclusion of amplitude fluctuations, because the superfluid density $\rho_{s}$ appears as a coefficient in the quadratic fluctuation action in the long-wavelength approximation [10,11,13], thus corrections to the superfluid density mean an account of higher-order terms in powers of the fluctuation coordinates. However, the saddle-point gap parameter $\Delta$ and the chemical potentials $\mu, \zeta$ will change when including amplitude fluctuations. The resulting temperature-imbalance phase diagram, obtained in [14], is reproduced in Fig. 1 at binding energy $E_{b}=0.1 E_{F}$. Figure 1(a) shows the results without amplitude fluctuations, while Fig. 1(b) represents the phase diagram obtained after including these fluctuations.

At high temperature and/or high imbalance, we have the normal phase, where $\Delta=0$ and $\rho_{s}=0$. When $\Delta \neq 0$, pairing takes place and a quasicondensate [42] is formed. The temperature below which the quasicondensate is formed will be denoted by $T_{c}^{*}$. This quasicondensate is superfluid below the BKT temperature $T_{\mathrm{BKT}}$, but above the BKT temperature, vortex-antivortex pairs unbind and phase coherence is lost, so that $\rho_{s}=0$ and we have pairing without coherence. There is a region in the phase diagram, indicated as "phase-separated state,"where the combination of the thermodynamic parameters does not lead to a joint solution of Eqs. (5)-(8). As a result, the system separates in a nearly balanced quasicondensate and a normal part that carries the excess particles.

One of the first observations of a quasicondensate was made for a monolayer of hydrogen atoms adsorbed on a helium film [45]. It is a challenging problem how to experimentally determine the quasicondensate phase for a trapped quasi-2D gas of cold fermionic atoms. To verify different phases of those systems, the phase separation in a trap seems to be the most effective method, especially in the presence of population imbalance. In general, the critical temperatures $T_{\mathrm{BKT}}$ and $T_{c}^{*}$ differ from each other. Therefore, a shell of noncoherent quasicondensate can appear between the superfluid core and the surrounding normal phase for a quasi-2D Fermi gas in a trap.

When cold fermions are confined to a trap, different phases are spatially separated from each other. According to the phase diagrams, for 2D fermions in a trap there can be, in general, two phase boundaries. The first phase boundary separates the superfluid state (where the superfluid pair density $\rho_{s} \neq 0$ ) and the noncoherent quasicondensate (where $\rho_{s}=0$ but $\Delta \neq 0$ ). This phase boundary is determined from the joint solution of the gap and number equations, generalized to the imbalance case, together with Eq. (8) for $T=T_{\mathrm{BKT}}$. The second phase boundary separates the noncoherent quasicondensate and 
the normal state (where $\Delta=0$ ). This phase boundary is determined by the condition $T=T_{c}^{*}$. So, for a 2D trapped Fermi gas, the superfluid core is surrounded by the noncoherent quasicondensate shell. The quasicondensate is surrounded by the normal state. For a nonzero population imbalance, there is a jump of the gap parameter from a finite value to zero at the phase boundary between the quasicondensate and the normal state. Thus the aforementioned phase transition can be of first order when the population imbalance $\delta n \neq 0$.

The BKT phase transition is not of first order. However, at $T=T_{\mathrm{BKT}}$, the superfluid density undergoes a jump [6] because $\rho_{s}$ remains finite as long as $T$ is lower than $T_{\mathrm{BKT}}$ while $\rho_{s}=0$ for $T>T_{\mathrm{BKT}}$. Therefore, there is a jump of the pair superfluid density at the phase boundary between the superfluid state and the noncoherent quasicondensate. On the contrary, the gap parameter as well as the total local fermion density at sufficiently low imbalance (including the balanced case $\zeta=0$ ) are continuous at the BKT phase boundary. This smooth

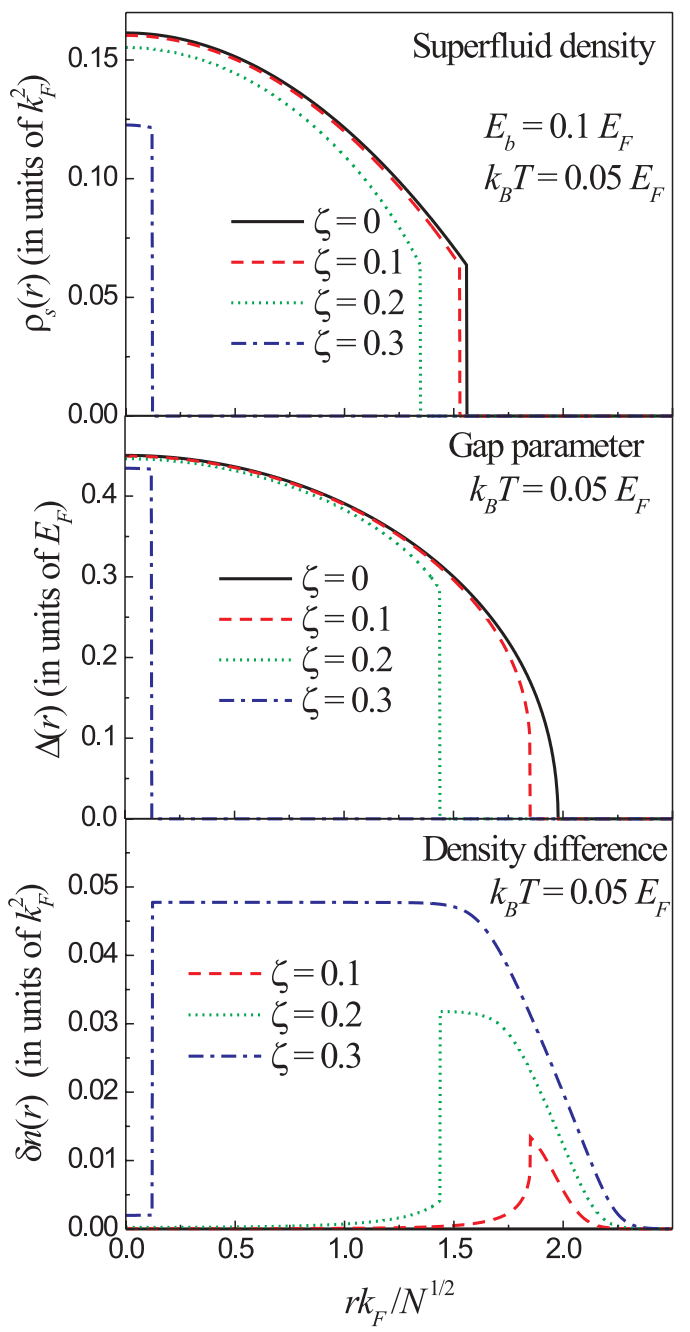

FIG. 2. (Color online) The pair superfluid density $\rho_{s}(r)$ (in units of the squared Fermi wave vector, $k_{F}^{2}$ ), the gap parameter $\Delta(r)$ (in units of $E_{F}$ ), and the local density difference $\delta n(r)$ (in units of $k_{F}^{2}$ ) for imbalanced trapped fermions in 2D, as a function of the distance $r$ to the center of the trap, are shown at binding energy $E_{b}=0.1 E_{F}$ and temperature $T=0.05 E_{F} / k_{B}$ for different values of the chemical potential imbalance. behavior of the density is in line with the results of Ref. [46], where no discontinuity of the local density is found at the BKT phase boundary for a trapped Bose gas in 2D.

The radius of the superfluid core $R_{\mathrm{BKT}}$ and the outer radius of the quasicondensate $R_{c}^{*}$ decrease with increasing temperature and with decreasing binding energy. For a balanced gas and for a relatively small imbalance, $R_{\mathrm{BKT}}<R_{c}^{*}$. For a sufficiently high imbalance, the superfluid phase transition is of first order, and those two radii coincide. We see from Fig. 2 that the distribution of the density imbalance $\delta n$ exhibits a pronounced jump at $r=R_{c}^{*}$, i.e., at the boundary between the quasicondensate and the normal phase. The local density difference achieves its maximum at the phase boundary between the quasicondensate and the normal state. In the noncoherent quasicondensate shell, the local density difference $\delta n$ strongly falls off with decreasing radius. The density difference in the superfluid core and in the quasicondensate is much lower than in the surrounding normal phase. In this connection, in the further analysis of collective excitations, we approximately consider the Fermi gas in the superfluid core and in the quasicondensate shell as a balanced gas.

\section{EQUILIBRIUM CONFIGURATION OF THE TRAPPED IMBALANCED FERMI GAS}

\section{A. Polytropic parameters obtained from the microscopic description}

The microscopic equations of state for trapped fermions can be determined numerically from the equilibrium distributions of the density and pressure in a trap obtained within the approach described above. These equations of state can in turn be used in combination with a hydrodynamic description to calculate numerically the dispersion relation for the collective modes of the multicomponent trapped Fermi gas. However, when the equations of state have the form of a polytropic law relating pressure to density, $P(n)=C n^{\gamma+1}$, the collective mode dispersion can be solved analytically.

In Refs. [22,23], an analytic solution in terms of hypergeometric functions is obtained for the amplitudes of the density oscillations for $T=0$ using the Gibbs-Duhem relation [47,48]. Heiselberg [24] derived analytic expressions for collective modes for trapped gases in $3 \mathrm{D}$ at finite temperatures assuming a polytropic equation of state. Later, the simulation of the equation of state using a polytropic law was used for the analysis of collective excitations of balanced trapped cold fermions in three-dimensional traps [25-32]. In the present work, the method inspired by Ref. [24] is applied to trapped and imbalanced Fermi gases in 2D.

The approach that we will follow consists, therefore, in fitting a polytropic law to the numerically obtained equations of state of the different phases. The resulting polytropic exponents $\gamma$ and $C$ are then used as input for the analytic results. The calculation of the collective modes using the fitted polytropic laws is performed in the next section; in this subsection, we describe the fitting procedure.

We consider the case in which the thermal energy $k_{B} T$ is substantially lower than the Fermi energy. Therefore, we can approximately treat the superfluid core and the noncoherent quasicondensate shell as spin-balanced subsystems. As far 
as the equations of state for the quasicondensate with and without phase coherence are the same, the quasicondensate phase in the core with the radius $R_{b} \equiv R_{c}^{*}$ is approximately suggested to be a fully balanced phase. Moreover, since the coherent and phase-incoherent quasicondensate phases cannot be distinguished from the point of view of the equation of state, our current analysis will not involve $R_{\mathrm{BKT}}$, and will treat the coherent and incoherent quasicondensate phases as a single phase as far as the density distribution is concerned. So, in total our description deals with three components of the confined system of cold atoms: the quasicondensate component $(S)$ and the spin-up $(\uparrow)$ and spin-down $(\downarrow)$ normal components. The numerical microscopic dependences of the pressure versus density $P_{j}\left(n_{j}\right)$ for each phase are fitted using the polytropic law $P_{j}^{(\mathrm{pt})}\left(n_{j}\right)$,

$$
P_{j}\left(n_{j}\right) \approx P_{j}^{(\mathrm{pt})}\left(n_{j}\right)=C_{j} n_{j}^{\gamma_{j}+1},
$$

where $P_{j}$ is the pressure, $n_{j}$ is the particle density, and $\gamma_{j}$ is the polytropic index for the $j$ th phase. Here $j=S, \uparrow, \downarrow$ indicates the component.

The distribution of the density in a parabolic trapping potential characterized by the confinement frequency $\omega_{0}$,

$$
U(r)=\frac{1}{4} \omega_{0}^{2} r^{2},
$$

is explicitly found from the equation of state (10) and thermodynamic laws, similarly to Ref. [24]. The result is

$$
n_{j}^{(\mathrm{pt})}(r)=\left[\frac{R_{j}^{2} \gamma_{j}}{4 N\left(\gamma_{j}+1\right) C_{j}}\left(1-\frac{r^{2}}{R_{j}^{2}}\right)\right]^{\frac{1}{\gamma_{j}}} \Theta\left(R_{j}-r\right),
$$

where $N$ is the total number of fermions in the trap, $R_{j}$ is the outer radius for the distribution for each component, and $\Theta\left(R_{j}-r\right)$ is the Heaviside step function.

The indices $\gamma_{j}$ of the polytropic equations of state for each (quasicondensate and normal) component can be determined through analytic fits for dependences $P_{j}\left(n_{j}\right)$ calculated numerically taking into account amplitude and phase fluctuations. In practice, the optimal values of the polytropic parameters $C_{j}, \gamma_{j}$ are found using minimization of the mean-square deviations:

$$
\begin{gathered}
\Delta P_{S} \equiv \int_{0}^{R_{b}}\left[P_{S}(r)-C_{S} n_{S}^{\gamma_{S}+1}(r)\right]^{2} r d r, \\
\Delta P_{p} \equiv \int_{R_{b}}^{\infty}\left[P_{p}(r)-C_{p} n_{p}^{\gamma_{p}+1}(r)\right]^{2} r d r \quad \text { for } p=\uparrow, \downarrow,
\end{gathered}
$$

where $P_{j}(r)$ and $n_{j}(r)$ are the distributions of the pressure and of the density calculated within the microscopic approach described in Sec. II.

For the quasicondensate, the resulting optimal values of the parameters $C_{S}$ and $\gamma_{S}$ at finite temperatures differ only slightly from their zero-temperature limits $C_{S}^{(0)}=\pi$ and $\gamma_{S}^{(0)}=1$ determined analytically in Ref. [23]. On the contrary, polytropic parameters for the normal phase at finite temperatures are very different from their values at $T=$ 0 and depend strongly on the total population imbalance $Q \equiv\left(N_{\uparrow}-N_{\downarrow}\right) / N$ as shown in Fig. 3 and in Table I. This distinction is due to the fact that at sufficiently low densities, the normal phase at a finite temperature is very different from the normal phase at $T=0$. Namely, even at relatively low

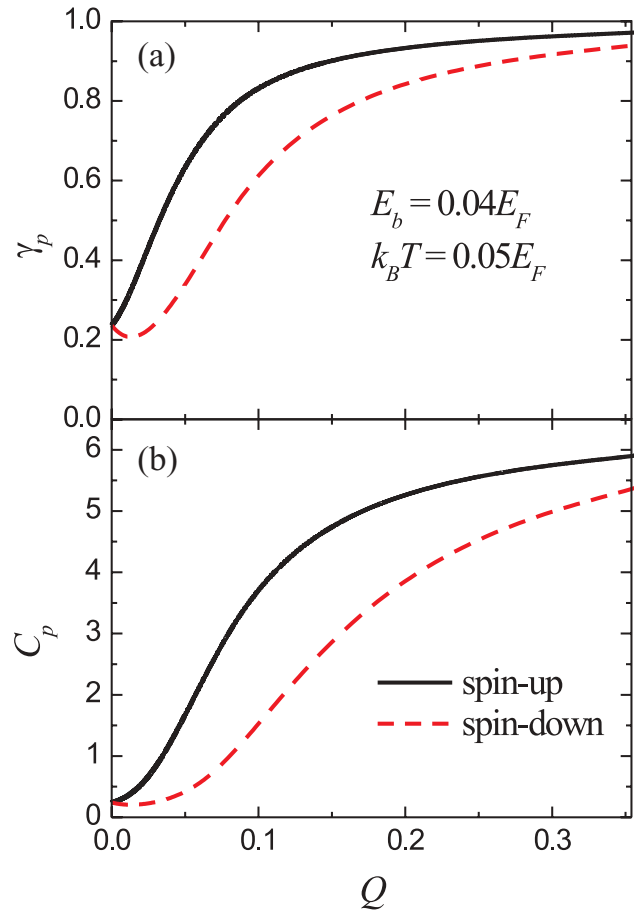

FIG. 3. (Color online) The parameters of the polytropic equation of state $C_{p}$ (a) and $\gamma_{p}$ (b) $(p=\uparrow, \downarrow)$ for an imbalanced Fermi gas in $2 \mathrm{D}$, obtained by fitting to the path-integral calculation, are shown as a function of the relative population imbalance $Q$, up to the maximum imbalance that can sustain a superfluid core, which for the given parameters $\left(E_{b}=0.04 E_{F}\right.$ and $\left.T=0.05 E_{F} / k_{B}\right)$ occurs at $Q \approx 0.354$.

temperature $T=0.05 E_{F} / k_{B}$, the chemical potential of the normal phase at $r=R_{b}$ is negative in the balanced case. Therefore, in the balanced case and at a low imbalance, the coefficients $C_{p}$ and $\gamma_{p}$ for $p=\uparrow, \downarrow$ are much smaller than their zero-temperature values from Ref. [23], $C_{p}^{(0)}=2 \pi$ and $\gamma_{p}^{(0)}=1$. With increasing imbalance, the density of the majority (e.g., spin-up) component increases monotonically, and the density of the minority (spin-down) component lowers slightly and then increases. The corresponding behavior of the polytropic parameters is clearly seen in Fig. 3. With further rising of $Q$, parameters $C_{p}$ and $\gamma_{p}$ at sufficiently low temperatures become, respectively, equal to $C_{p}^{(0)}$ and $\gamma_{p}^{(0)}$. As seen from Table I, for sufficiently weak coupling (when the superfluid core vanishes), the polytropic indices for the normal phase become essentially independent of the coupling strength.

\section{B. Equilibrium configuration of superfluid and normal components}

To calculate the oscillation frequencies of the collective modes, it is necessary to know the equilibrium configuration around which small oscillations will take place. The equilibrium configuration is characterized by seven independent parameters $\left(R_{S}, R_{\uparrow}, R_{\downarrow}, N_{S}, N_{\uparrow}, N_{\downarrow}, R_{b}\right)$, where $N_{j}$ and $R_{j}$ with $j=S, \uparrow, \downarrow$ are the number of fermions and the radii of the density for the $j$ th component, as in Eq. (12). $R_{b}$ is the outer radius of the quasicondensate $R_{b}=R_{c}^{*}$. The complete set of parameters for the equilibrium configuration are determined using the boundary conditions for the pressure and the 
TABLE I. Parameters for a trapped imbalanced fermion gas in 2D.

\begin{tabular}{|c|c|c|c|c|c|c|}
\hline \multirow{2}{*}{$\frac{\zeta=0, Q=0}{E_{b} / E_{F}}$} & \multicolumn{3}{|c|}{$T=0.05 E_{F} / k_{B}$} & \multicolumn{3}{|c|}{$T=0.1 E_{F} / k_{B}$} \\
\hline & 0.04 & 0.1 & 0.5 & 0.04 & 0.1 & 0.5 \\
\hline$C_{\uparrow}, C_{\downarrow}$ & 0.24176 & 0.08613 & 0.05253 & 1.24290 & 0.31185 & 0.10999 \\
\hline$\gamma_{\uparrow}, \gamma_{\downarrow}$ & 0.23624 & 0.06816 & 0.00438 & 0.48806 & 0.18177 & 0.01036 \\
\hline$\lambda_{\uparrow}, \lambda_{\downarrow}$ & 0.00728 & 0.00166 & $0.17 \times 10^{-4}$ & 0.09053 & 0.02050 & 0.00092 \\
\hline$\zeta=0.1 E_{F}$ & \multicolumn{3}{|c|}{$T=0.05 E_{F} / k_{B}$} & \multicolumn{3}{|c|}{$T=0.1 E_{F} / k_{B}$} \\
\hline$E_{b} / E_{F}$ & 0.04 & 0.1 & 0.5 & 0.04 & 0.1 & 0.5 \\
\hline$Q$ & 0.0891 & 0.0316 & $2.9 \times 10^{-4}$ & 0.1672 & 0.0847 & 0.0056 \\
\hline$\tilde{C}_{\uparrow}$ & 3.35710 & 0.96833 & 0.05314 & 2.94740 & 0.84454 & 0.12621 \\
\hline$C_{\downarrow}$ & 1.22670 & 0.10003 & 0.05071 & 1.74920 & 0.28844 & 0.10433 \\
\hline$\gamma_{\uparrow}$ & 0.80515 & 0.51047 & 0.00583 & 0.72324 & 0.39450 & 0.02848 \\
\hline$\gamma_{\downarrow}$ & 0.56242 & 0.09016 & 0.00107 & 0.57642 & 0.16686 & 0.00420 \\
\hline$\lambda_{\uparrow}$ & 0.09744 & 0.02520 & 0.00013 & 0.24281 & 0.06180 & 0.00260 \\
\hline$\lambda_{\downarrow}$ & 0.03101 & 0.00226 & $2.4 \times 10^{-6}$ & 0.12816 & 0.01843 & $3.71 \times 10^{-4}$ \\
\hline$\zeta=0.2 E_{F}$ & \multicolumn{3}{|c|}{$T=0.05 E_{F} / k_{B}$} & \multicolumn{3}{|c|}{$T=0.1 E_{F} / k_{B}$} \\
\hline$E_{b} / E_{F}$ & 0.04 & 0.1 & 0.5 & 0.04 & 0.1 & 0.5 \\
\hline$Q$ & 0.3816 & 0.1818 & 0.00236 & 0.3728 & 0.2617 & 0.0180 \\
\hline$C_{\uparrow}$ & 5.95190 & 4.73460 & 0.07573 & 5.12260 & 3.24170 & 0.18690 \\
\hline$C_{\downarrow}$ & 5.44790 & 1.27420 & 0.05118 & 3.87470 & 0.96250 & 0.10245 \\
\hline$\gamma_{\uparrow}$ & 0.97489 & 0.90071 & 0.05004 & 0.90481 & 0.75196 & 0.08929 \\
\hline$\gamma_{\downarrow}$ & 0.94427 & 0.57097 & 0.00159 & 0.80851 & 0.42556 & 0.00226 \\
\hline$\lambda_{\uparrow}$ & 0.69080 & 0.20637 & 0.00118 & 0.68641 & 0.28094 & 0.00886 \\
\hline$\lambda_{\downarrow}$ & 0.30920 & 0.03194 & $4.5 \times 10^{-7}$ & 0.31359 & 0.06964 & $2.0 \times 10^{-4}$ \\
\hline
\end{tabular}

chemical potential. The boundary condition for pressure at equilibrium is

$$
\left.\left(P_{S}-P_{\uparrow}-P_{\downarrow}\right)\right|_{r=R_{b}}=0 .
$$

In the present analysis, the surface tension of this interface is neglected.

Let us consider the scheme of chemical potentials of the system shown in Fig. 4. The coordinate-dependent effective chemical potentials are related to the true chemical potentials $\mu_{j, 0}$ as

$$
\mu_{j}(r)=\mu_{j, 0}-U(r)
$$

The averaged chemical potential of spin-up and spin-down normal components is $\mu_{0}=\left(\mu_{\uparrow, 0}+\mu_{\downarrow, 0}\right) / 2$. The chemical potential imbalance for the normal components is $\zeta=\left(\mu_{\uparrow, 0}-\right.$ $\left.\mu_{\downarrow, 0}\right) / 2$.

When a pair is formed or destroyed, the averaged chemical potential must be conserved. Therefore, we have the condition

$$
\mu_{\uparrow, 0}+\mu_{\downarrow, 0}=2 \mu_{S, 0} .
$$

Equation (16) automatically provides the boundary condition for the effective chemical potentials

$$
\mu_{\uparrow}\left(R_{b}\right)+\mu_{\downarrow}\left(R_{b}\right)-2 \mu_{S}\left(R_{b}\right)=0 .
$$

Equations (14) and (17) express equilibrium between the pair quasicondensate on the inside of the boundary at $R_{b}$ and the two-component normal gas on the outside of the interface at $R_{b}$.

Since there are seven parameters describing the equilibrium configuration, another five equations are necessary in addition to the aforementioned two boundary conditions. These equations are provided by three normalization conditions for each component of the density,

$$
\begin{gathered}
2 \pi \int_{0}^{R_{b}} n_{S}(r) r d r=N_{S}, \\
2 \pi \int_{R_{b}}^{R_{p}} n_{p}(r) r d r=N_{p} \quad \text { for } \quad p=\uparrow, \downarrow,
\end{gathered}
$$

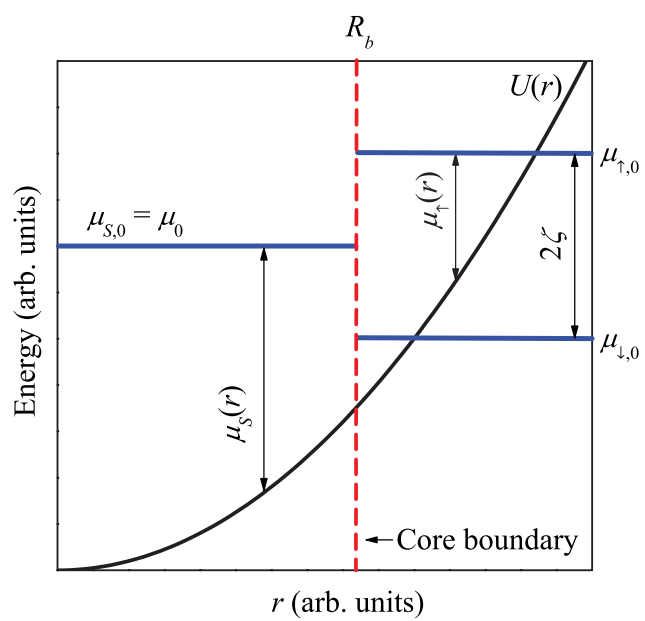

FIG. 4. (Color online) Chemical potentials schematically shown for trapped fermions in 2D. The parabolic curve is the trapping potential. The vertical dashed line indicates the boundary of the quasicondensate core. 
completed with two normalization conditions,

$$
\begin{gathered}
\lambda_{\uparrow}+\lambda_{\downarrow}+\lambda_{S}=1, \\
\lambda_{\uparrow}-\lambda_{\downarrow}=Q_{p} .
\end{gathered}
$$

Here, $\lambda_{j} \equiv N_{j} / N$ are the relative populations of three components (the quasicondensate and the spin-up and spindown normal components), and $Q_{p}$ is the relative population imbalance within the polytropic model (in which the core is nonpolarized). The values of $\lambda_{j}$ determined from the microscopic calculation as described above are listed in Table I.

As far as the quasicondensate core at low but finite temperatures is slightly polarized, the total relative population imbalance $Q$ determined from the microscopic calculation can differ from $Q_{p}$ related only to the normal components outside the core. A replacement of $Q$ by $Q_{p}$ can lead to only a scaling of the obtained eigenfrequency spectra along the $Q$ axis without any other change of eigenfrequencies. Here, we prefer $Q$ rather than $Q_{p}$ to be used as a measure of imbalance. The values of $Q$ for several temperatures and coupling strengths are also represented in Table I.

Using the analytic coordinate dependence of the density (12) in (18) and (19), the integrals (18) and (19) are calculated straightforwardly. Hence we arrive at the analytic normalization conditions

$$
\begin{gathered}
\pi\left(\frac{1}{4 C_{S} N}\right)^{\frac{1}{\gamma_{S}}}\left(\frac{\gamma_{S}}{\gamma_{S}+1}\right)^{\frac{1}{\gamma_{S}}+1}\left[R_{S}^{2\left(\frac{1}{\gamma_{S}}+1\right)}-\left(R_{S}^{2}-R_{b}^{2}\right)^{\frac{1}{\gamma_{S}}+1}\right] \\
=N_{S}, \\
\pi\left(\frac{1}{4 C_{p} N}\right)^{\frac{1}{\gamma_{p}}}\left(\frac{\gamma_{p}}{\gamma_{p}+1}\right)^{\frac{1}{\gamma_{p}}+1}\left(R_{p}^{2}-R_{b}^{2}\right)^{\frac{1}{\gamma_{p}}+1}=N_{p} \text { for } \\
p=\uparrow, \downarrow .
\end{gathered}
$$

The complete set of equations for the analytic approximation of the equilibrium density distribution of trapped fermions in 2D consists of Eqs. (14), (17), and (20)-(23). For the model system obeying the polytropic equations of state, the specific radii entering the equations for collective modes can be expressed analytically through the relative populations $\lambda_{j}$. The boundary condition for the pressure (14) gives the explicit result for $R_{S}$ :

$$
R_{S}=\left[4 N C_{S} \frac{\gamma_{S}+1}{\gamma_{S}}\left(\frac{1}{4 C_{S} \pi}\right)^{\frac{\gamma_{S}}{\gamma_{S}+1}}\right]^{1 / 2} .
$$

Applying this result to the normalization conditions, we find the other specific radii:

$$
\begin{gathered}
R_{b}=\left[4 N C_{S}\left(1+\frac{1}{\gamma_{S}}\right)\left(\frac{1}{4 C_{S} \pi}\right)^{\frac{\gamma_{S}}{1+\gamma_{S}}}\left[1-\left(1-\lambda_{S}\right)^{\frac{\gamma_{S}}{1+\gamma_{S}}}\right]\right]^{1 / 2}, \\
R_{p}=\left[R_{b}^{2}+4 N C_{p}\left(1+\frac{1}{\gamma_{p}}\right)\left(\frac{\lambda_{p}}{4 C_{p} \pi}\right)^{\frac{\gamma_{p}}{1+\gamma_{p}}}\right]^{1 / 2} \text { for } p=\uparrow, \downarrow .
\end{gathered}
$$

In the zero-temperature limit, these expressions reproduce the results of Ref. [23].

In Fig. 5, we compare the density distributions simulated by (12) obtained using the polytropic law (10) to those calculated using the microscopic approach described in Sec. II at

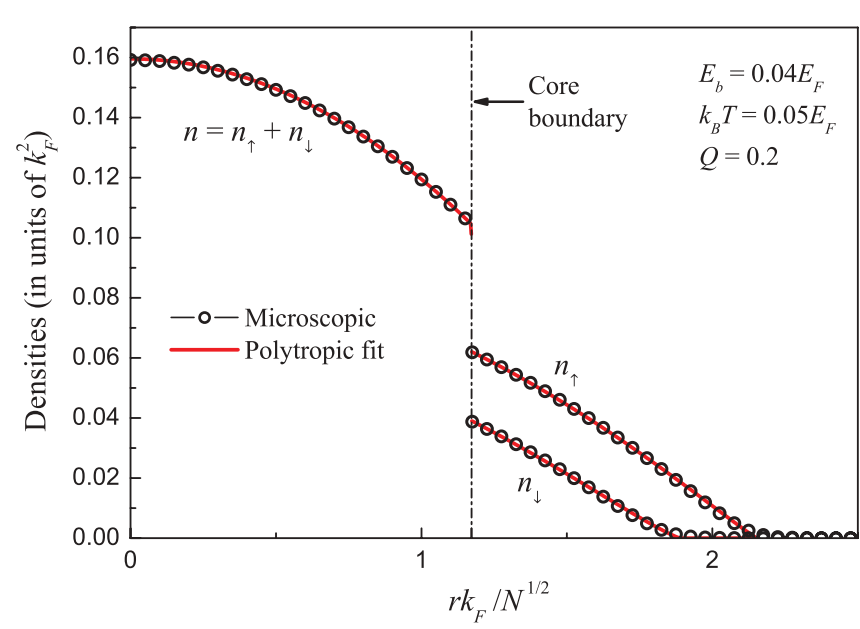

FIG. 5. (Color online) Densities of the spin-up and spin-down components (in units of $k_{F}^{2}$ ) for imbalanced trapped fermions in 2D, as a function of the distance $r$ to the center of the trap, at binding energy $E_{b}=0.04 E_{F}$, temperature $T=0.05 E_{F} / k_{B}$, and relative population imbalance $Q=0.2$. The density distributions obtained using polytropic equations of state are shown by curves. The results of the microscopic calculation are shown by open circles.

binding energy $E_{b}=0.04 E_{F}$, temperature $T=0.05 E_{F} / k_{B}$, and relative population imbalance $Q=0.2$. We can see that the density distributions for a polytropic system with an appropriate choice of the polytropic parameters fit well to the microscopic density profiles.

\section{COLLECTIVE EXCITATIONS}

\section{A. Approach}

Having obtained the parameters for the polytropic equations of state from the microscopic theory, and having determined the equilibrium configuration, we can investigate small-amplitude collective oscillations around the equilibrium configuration using the hydrodynamic approach. With a linear expansion in powers of small vibrational coordinates and velocities, the linearized Euler and continuity equations for each component are

$$
\begin{gathered}
\frac{1}{2} n_{j}(r) \frac{\partial \mathbf{v}_{j}(\mathbf{r}, t)}{\partial t}=-\nabla\left[\delta P_{j}(\mathbf{r}, t)\right]-\delta n_{j}(\mathbf{r}, t) \nabla U(r), \\
\frac{\partial\left(\delta n_{j}(\mathbf{r}, t)\right)}{\partial t}=-\nabla \cdot\left[n_{j}(r) \mathbf{v}_{j}(\mathbf{r}, t)\right],
\end{gathered}
$$

where $\mathbf{v}_{j}(\mathbf{r}, t)$ is the velocity, $\delta P_{j}(\mathbf{r}, t)$ is the deviation of the pressure from its equilibrium value $P_{j}(r), \delta n_{j}(\mathbf{r}, t)$ is the oscillation amplitude of the density about its equilibrium distribution $n_{j}(r)$, and $U(r)$ is the trapping potential.

According to the two-fluid Landau hydrodynamics for superfluids [49], also the continuity equation for entropy must be taken into account:

$$
\frac{\partial\left(\delta s_{j}(\mathbf{r}, t)\right)}{\partial t}=-\nabla \cdot\left[s_{j}(r) \mathbf{v}_{j, n}(\mathbf{r}, t)\right],
$$

where $s_{j}(r)$ is the equilibrium entropy density, $\delta s_{j}(\mathbf{r}, t)$ is the entropy density oscillation amplitude, and $\mathbf{v}_{j, n}(\mathbf{r}, t)$ is the velocity of the normal fraction for the $j$ th component. However, in the present approach, normal and superfluid 
fractions are suggested to be completely spatially separated, so that $\mathbf{v}_{j, n}=\mathbf{v}_{j}$ for the normal components and $\mathbf{v}_{j, n}=0$ for the superfluid. As a result, the oscillations of entropy $\delta s_{j}(\mathbf{r}, t)$ for each normal component are determined by the motion of the whole component and give no feedback to the Euler and continuity equations. As far as the polytropic equation of state is given, Eqs. (27) and (28) constitute a complete and closed set of equations for oscillation amplitudes. In this connection, the continuity equation for entropy is not involved in the resulting set of equations for oscillation eigenfrequencies. Furthermore, it is often suggested that the oscillation motion of trapped cold atoms is adiabatic, and therefore the conservation of entropy is automatically fulfilled [50].

A partial solution of the above equations with definite frequency $\omega$ and angular momentum $l$ can be written in the polar coordinates $\mathbf{r}=(r, \varphi)$ :

$$
\delta n_{j}(\mathbf{r}, t \mid l, \omega)=\delta n_{j}(r, l, \omega) e^{i l \varphi-i \omega t} .
$$

The spatial dependence of the vibrational amplitudes of the density with the polytropic equation of state (10) is the joint solution of the Euler and continuity equations. This solution is expressed through hypergeometric functions (see the derivation in Appendix B):

$$
\begin{aligned}
\delta n_{S}(r, l, \omega)=A_{S} r^{l} F\left(a_{S}, b_{S} ; l+1 ; \frac{r^{2}}{R_{S}^{2}}\right), & \\
\delta n_{p}(r, l, \omega)= & A_{p} r^{l}\left(1-\frac{r^{2}}{R_{p}^{2}}\right)^{\frac{1}{\gamma_{p}}-1} \\
& \times F\left(l+1-a_{p}, l+1-b_{p} ; \frac{1}{\gamma_{p}} ; 1-\frac{r^{2}}{R_{p}^{2}}\right),
\end{aligned}
$$

with constants $A_{j}$ (for the quasicondensate $j=S$ and for the normal phase $j \equiv p$ with $p=\uparrow, \downarrow)$ and parameters

$$
\begin{aligned}
& a_{j}=\frac{1}{2 \gamma_{j}}\left((l+2) \gamma_{j}-1+\sqrt{1+\gamma_{j}^{2} l^{2}+2 \gamma_{j} \frac{\omega^{2}}{\omega_{0}^{2}}}\right), \\
& b_{j}=\frac{1}{2 \gamma_{j}}\left((l+2) \gamma_{j}-1-\sqrt{1+\gamma_{j}^{2} l^{2}+2 \gamma_{j} \frac{\omega^{2}}{\omega_{0}^{2}}}\right) .
\end{aligned}
$$

The eigenfrequencies and the amplitudes of the collective excitations are determined using the boundary conditions for the pressure, the chemical potential, and the current flow. The boundary condition for the pressure is

$$
\left.\left(P_{S}-P_{\uparrow}-P_{\downarrow}\right)\right|_{\xi}=0,
$$

where $\xi=R_{b}+\delta R(t)$ describes the interface between the quasicondensate and the normal phase.

The chemical potential must be continuous at the phase boundary:

$$
\left.\left(2 \mu_{S}-\mu_{\uparrow}-\mu_{\downarrow}\right)\right|_{\xi}=0 .
$$

Also, we apply the continuity equation at the boundary [22] suggesting that particles may pass over between the quasicondensate and the normal phase,

$$
\mathbf{e}_{\xi} \cdot\left(2 \mathbf{v}_{p} n_{p}-\mathbf{v}_{S} n_{S}\right)_{\xi}=\left(2 n_{p}-n_{S}\right)_{\xi} \frac{\partial(\delta R)}{\partial t} \quad \text { for } \quad p=\uparrow, \downarrow,
$$

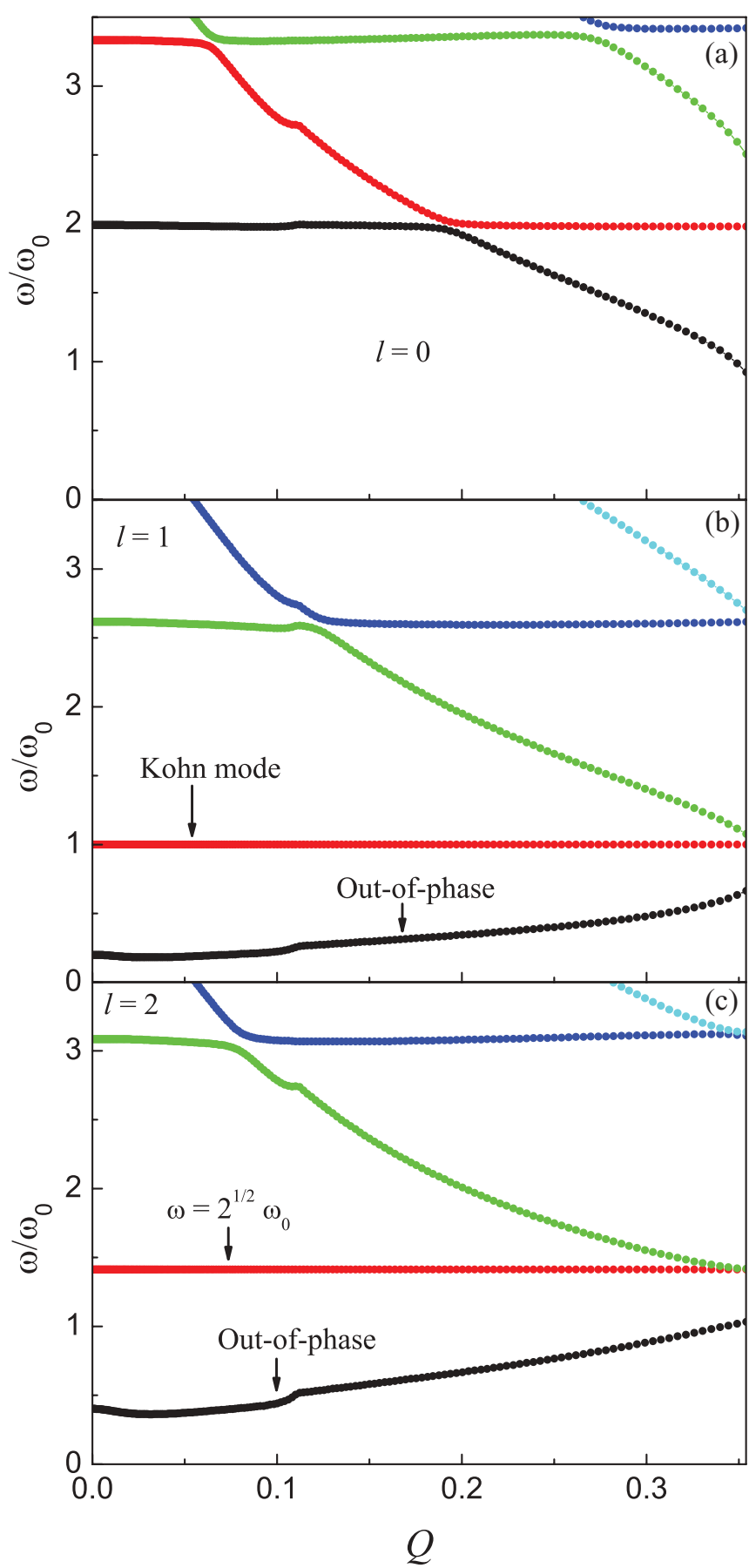

FIG. 6. (Color online) Eigenfrequencies of collective modes with $l=0$ (a), $l=1$ (b), and $l=2$ (c) for a trapped imbalanced Fermi gas in $2 \mathrm{D}$ at $E_{b}=0.04 E_{F}$ and $T=0.05 E_{F} / k_{B}$ as a function of the relative population imbalance $Q$. Different branches shown in the figure are related to different radial quantum numbers $k$ with one and the same angular momentum $l$. 
where $\mathbf{e}_{\xi}$ is the unit vector normal to the interface $\xi$, and $\delta R$ is the mechanical displacement of the interface.

The set of equations for collective modes consists of four equations for amplitudes $A(l, \omega), A_{\uparrow}(l, \omega), A_{\downarrow}(l, \omega)$ [which enter the solution (31) and (32)], and the amplitude of the mechanical displacement of the boundary $\delta R(l, \omega)$. These equations follow from four boundary conditions (35)-(37) and constitute a uniform linear set (see details in Appendix C). Eigenfrequencies of collective modes are then obtained from the condition that the determinant of this set of equations is equal to zero. The roots of the equation for eigenfrequencies are calculated numerically.

\section{B. Eigenfrequencies}

In Fig. 6, we plot several eigenfrequencies (for angular momentum $l=0,1,2$ and for different values of the radial quantum number $k$ ) of collective oscillations at $T=0.05 E_{F} / k_{B}$ and binding energy $E_{b}=0.04 E_{F}$. The frequencies are plotted as a function of the total population imbalance $Q$. Figure 7 shows eigenfrequencies at a binding energy $E_{b}=0.04 E_{F}$ and at fixed chemical potential imbalance $\zeta=0.1 E_{F}$ as a function of temperature.

There are two types of eigenfrequencies for the trapped fermion system: (i) eigenfrequencies attributed to the in-phase motion of all components (similarly to a single-component gas), and (ii) eigenfrequencies attributed to the out-of-phase motion of the inner core boundary and outer phase boundary of the trapped Fermi system.

The frequencies of the in-phase collective modes are slightly dependent on the population imbalance, but they can depend on temperature. The out-of-phase eigenfrequencies appear to be sensitive to the values of the parameters of the polytropic equation of state (10). This sensitivity is due to the fact that at low imbalance, the densities of both spin-up and spin-down normal components are very low. This is of small significance for the in-phase motion, but the out-ofphase motion depends substantially on the parameters of all components, despite a low weight of the normal components.

In general, the roots of the dispersion equation for frequencies of collective oscillations can be found only numerically. There are, however, some eigenfrequencies that are analytically exact. First, at any imbalance or temperature that allows for the existence of a quasicondensate-normal interface, there is a dipole mode $(l=1)$ with frequency exactly $\omega=\omega_{0}$, as follows from the generalized Kohn theorem [51] [see Figs. 6(b) and 7(b)]. Second, the quadrupole $(l=2)$ mode exists with frequency $\omega=\sqrt{2} \omega_{0}$ independent of the equation of state $[26,48]$. The breathing mode with the zero-temperature frequency $\omega=2 \omega_{0}$ [23] depends only slightly on temperature. However, it is not exactly equal to $2 \omega_{0}$ at nonzero temperatures.

An important difference between the eigenfrequencies of the trapped Fermi gas at finite temperatures and in the limit of $T=0$ [23] is the fact that with decreasing imbalance, low-lying eigenfrequencies corresponding to the out-of-phase mode (for $l \neq 0$ ) tend to a nonzero value in the balanced limit $Q=0$. On the contrary, at zero temperature, the frequencies of these out-of-phase collective modes tend to zero at a vanishing population imbalance. When temperature goes to zero, the eigenfrequencies of collective oscillations calculated in the

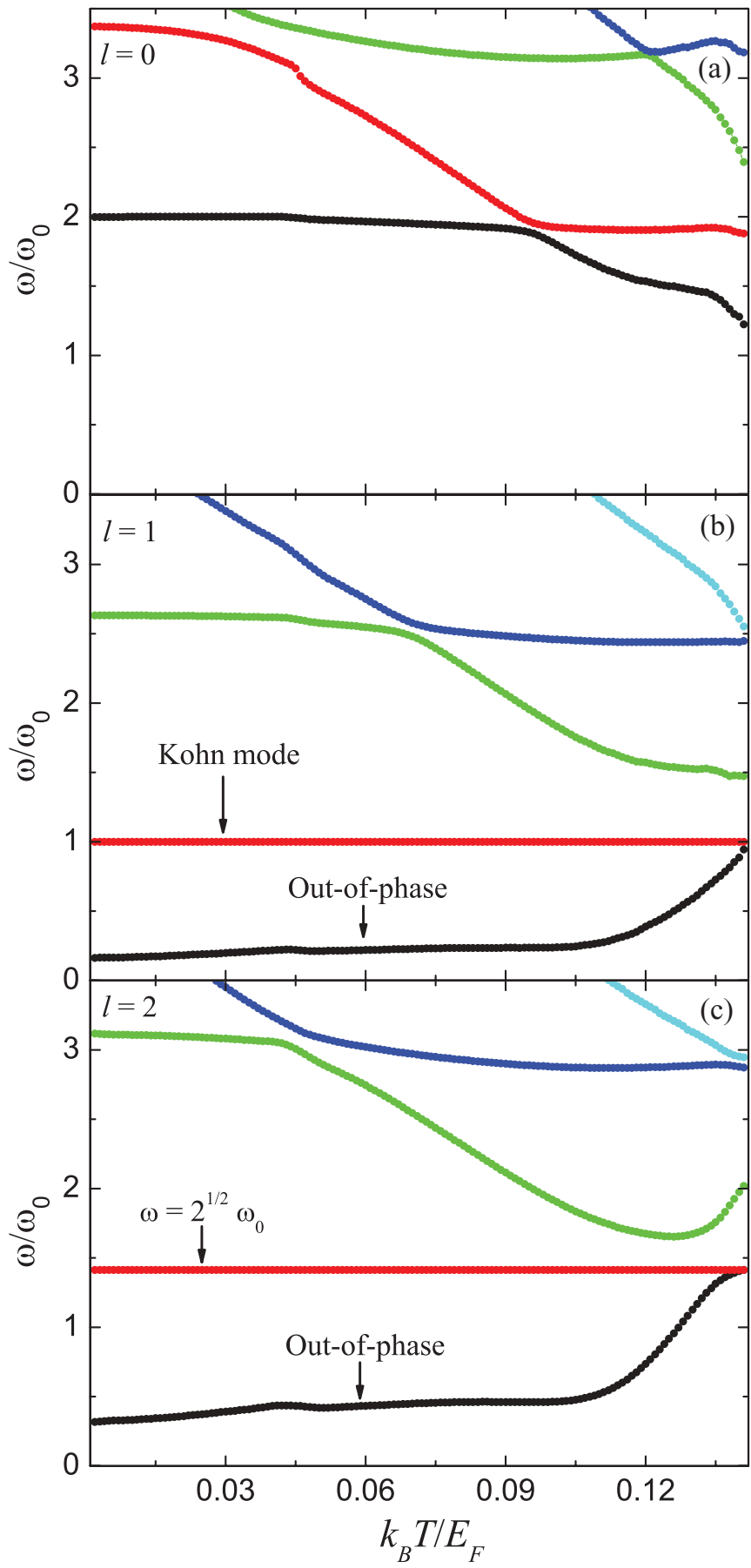

FIG. 7. (Color online) Eigenfrequencies of collective modes with $l=0$ (a), $l=1$ (b), and $l=2$ (c) for a trapped imbalanced Fermi gas in $2 \mathrm{D}$ at $E_{b}=0.04 E_{F}$ and chemical potential imbalance $\zeta=0.1 E_{F}$ as a function of temperature, up to the critical temperature $T_{c}^{*}$, which for these values of parameters is $T_{c}^{*} \approx 0.14 E_{F} / k_{B}$.

present work explicitly turn to the results obtained for $T=0$ in Ref. [23].

\section{CONCLUSIONS}

We have investigated phase diagrams and density distributions for a trapped Fermi gas in 2D with $s$-wave pairing, taking 
into account both phase and amplitude fluctuations and the BKT mechanism of the superfluid phase transition. The equation for the BKT transition temperature, the number equations, and the generalized gap equation are solved jointly. The present calculation confirms the often used assumption [14] that the BKT phase transition can be satisfactorily described taking into account phase fluctuations and neglecting amplitude fluctuations. However, to treat the complete phase diagram for imbalanced fermions, which includes the superfluid state, the normal state, the noncoherent quasicondensate, and the phaseseparated state, the incorporation of both phase and amplitude fluctuations is necessary [14]. The path-integral description, taking into account phase and amplitude fluctuations, offers a "microscopic" description from which we extract (through a fitting procedure) the parameters of a polytropic equation of state for the quasicondensate and the normal spin-up and spindown components of the system. This allows us to calculate the equilibrium configuration of the trapped superfluid-normal system analytically in the hydrodynamic approximation.

We have analyzed spectra of collective excitations of the Fermi gas in 2D confined to a parabolic trap within the hydrodynamic approach. This gives us the analytic dispersion equation for eigenfrequencies of collective excitations. The resulting eigenfrequencies reveal anticrossings between different modes. The obtained spectra of collective excitations drastically differ from those studied for the same system in the zero-temperature case [23]. In particular, collective excitations with low-lying frequencies attributed to the outof-phase motion tend to finite values at zero imbalance, as distinct from the corresponding out-of-phase modes at $T=0$. The behavior of frequencies of collective excitations when varying external parameters can be detected experimentally. It can provide information on the real equation of state of a trapped Fermi gas, and offers an experimental probe to detect superfluidity in Fermi gases in reduced dimensionality.

\section{ACKNOWLEDGMENTS}

Discussions with A. Lazarides are gratefully acknowledged. This work was supported by the Fonds voor Wetenschappelijk Onderzoek-Vlaanderen (FWO-V) projects G.0356.06, G.0370.09N, G.0180.09N, and G.0365.08.

\section{APPENDIX A: FLUCTUATION CONTRIBUTIONS TO THE FERMION DENSITY}

The fluctuation contributions to the averaged fermion density $n$ and to the population imbalance $\delta n$ are obtained using the same technique as in Refs. [37,38], replacing the Matsubara summations over bosonic fluctuation excitations by contour integrals with appropriate boundary conditions. The resulting expression for the fluctuation contribution to the fermion density is

$$
n_{\text {fluct }}=-\int \frac{d^{2} \mathbf{q}}{(2 \pi)^{2}} \frac{1}{\pi} \int_{-\infty}^{\infty} \frac{\operatorname{Im}[J(\mathbf{q}, \omega+i \delta)]}{e^{\beta \omega}-1} d \omega,
$$

where the function $J(\mathbf{q}, z)$ for a complex $z=\omega \pm i \delta$ is given by the formula

$$
\begin{aligned}
J(\mathbf{q}, z)= & \frac{1}{\Gamma(\mathbf{q}, z)}\left[M_{1,1}(\mathbf{q},-z) \frac{\partial M_{1,1}(\mathbf{q}, z)}{\partial \mu}\right. \\
& \left.-M_{1,2}(\mathbf{q},-z) \frac{\partial M_{1,2}(\mathbf{q}, z)}{\partial \mu}\right], \\
\Gamma(\mathbf{q}, z)= & M_{1,1}(\mathbf{q}, z) M_{1,1}(\mathbf{q},-z)-M_{1,2}^{2}(\mathbf{q}, z) .
\end{aligned}
$$

The fluctuation contribution to the population imbalance is determined by expressions similar to (A1) and (A2), with the replacement of $\frac{\partial M_{j, k}(\mathbf{q}, z)}{\partial \mu}$ to $\frac{\partial M_{j, k}(\mathbf{q}, z)}{\partial \zeta}$. The fluctuation matrix elements $M_{j, k}$ are given by

$$
\begin{aligned}
M_{1,1}(\mathbf{q}, z)= & \int \frac{d^{2} \mathbf{k}}{8 \pi^{2}} \frac{\sinh \left(\beta E_{\mathbf{k}}\right)}{\cosh \beta E_{\mathbf{k}}+\cosh \beta \zeta} \\
& \times \frac{1}{E_{\mathbf{k}}}\left(\frac{\left(z-E_{\mathbf{k}}+\xi_{\mathbf{k}+\mathbf{q}}\right)\left(E_{\mathbf{k}}+\xi_{\mathbf{k}}\right)}{\left(z-E_{\mathbf{k}}+E_{\mathbf{k}+\mathbf{q}}\right)\left(z-E_{\mathbf{k}}-E_{\mathbf{k}+\mathbf{q}}\right)}\right. \\
& \left.-\frac{\left(z+E_{\mathbf{k}}+\xi_{\mathbf{k}+\mathbf{q}}\right)\left(E_{\mathbf{k}}-\xi_{\mathbf{k}}\right)}{\left(z+E_{\mathbf{k}}-E_{\mathbf{k}+\mathbf{q}}\right)\left(z+E_{\mathbf{k}+\mathbf{q}}+E_{\mathbf{k}}\right)}\right)-\frac{1}{g}
\end{aligned}
$$

and

$$
\begin{aligned}
M_{1,2}(\mathbf{q}, z)= & -\int \frac{d^{2} \mathbf{k}}{8 \pi^{2}} \frac{\sinh \left(\beta E_{\mathbf{k}}\right)}{\cosh \beta E_{\mathbf{k}}+\cosh \beta \zeta} \\
& \times \frac{\Delta^{2}}{E_{\mathbf{k}}}\left(\frac{1}{\left(z-E_{\mathbf{k}}+E_{\mathbf{k}+\mathbf{q}}\right)\left(z-E_{\mathbf{k}}-E_{\mathbf{k}+\mathbf{q}}\right)}\right. \\
& \left.+\frac{1}{\left(z+E_{\mathbf{k}}-E_{\mathbf{k}+\mathbf{q}}\right)\left(z+E_{\mathbf{k}}+E_{\mathbf{k}+\mathbf{q}}\right)}\right)
\end{aligned}
$$

For the numerical calculation, we approximate the fluctuation contribution to the density by its interpolation between the weak-coupling limit (the mean-field approximation) and the strong-coupling limit (the approximation of a Bose-Fermi mixture, similar to Ref. [52]). In the strong-coupling limit, the matrix elements (A4) and (A5) can be asymptotically exactly replaced by their long-wavelength and low-energy expansions in powers of $q$ and $z$. The total fermion density within this approximation asymptotically tends to that obtained within the complete NSR-like approach $[37,38]$ both in the weakcoupling and strong-coupling limits.

\section{APPENDIX B: ANALYTIC FORM OF OSCILLATION AMPLITUDES}

The equation of motion for the density oscillations following from the Euler and continuity equations is

$$
m \frac{\partial^{2}}{\partial t^{2}}[\delta n(\mathbf{r}, t)]-\nabla^{2}[\delta P(\mathbf{r}, t)]-\nabla \cdot[\delta n(\mathbf{r}, t) \nabla U(r)]=0
$$

(for simplicity, here we omit indices numbering the components of the system). To find the eigenfrequencies, we consider the motion of a single mode,

$$
\mathbf{v}(\mathbf{r}, t)=\mathbf{v}(\mathbf{r}, \omega) e^{-i \omega t}, \quad \delta n(\mathbf{r}, t)=\delta n(\mathbf{r}, \omega) e^{-i \omega t},
$$


which results in the following equation in polar coordinates:

$$
\begin{gathered}
\nabla^{2}[\delta P(\mathbf{r}, \omega)]+m \omega_{0}^{2} r \frac{\partial[\delta n(\mathbf{r}, \omega)]}{\partial r} \\
+m\left(\omega^{2}+2 \omega_{0}^{2}\right) \delta n(\mathbf{r}, \omega)=0 .
\end{gathered}
$$

The modes with a definite angular momentum are determined as

$$
\delta n(\mathbf{r}, \omega)=\delta n(r, l, \omega) e^{i l \varphi} .
$$

This leads to the equation

$$
\begin{aligned}
& \left(\frac{\partial^{2}}{\partial r^{2}}+\frac{1}{r} \frac{\partial}{\partial r}-\frac{l^{2}}{r^{2}}\right)[\delta P(r, l, \omega)]+m \omega_{0}^{2} r \frac{\partial}{\partial r}[\delta n(r, l, \omega)] \\
& +m\left(\omega^{2}+2 \omega_{0}^{2}\right) \delta n(r, l, \omega)=0 .
\end{aligned}
$$

Further on, we consider the case in which the pressure $P(n)$ follows a polytropic law (10). Expanding $P(n+\delta n)$ in terms of $\delta n$ up to the first-order term leads to the following relation between the vibrational amplitudes of the pressure and of the density:

$$
\delta P(r, l, \omega)=(\gamma+1) C n^{\gamma} \delta n(r, l, \omega) .
$$

In equilibrium, the Euler equation is reduced to

$$
\nabla P(r)+n(r) \nabla U(r)=0 .
$$

The combination of Eq. (B6) with the polytropic law (10) provides the solution for the equilibrium density given by Eq. (12). In the close vicinity of the center of the trap, the solution of (B3) behaves as $\propto r^{ \pm l}$. Let us introduce the function $w(r)$

$$
\delta n(r, l, \omega) \equiv r^{l} w(r, l, \omega),
$$

and replace $r$ through the dimensionless variable $z$ as $r=$ $R \sqrt{z}$. Using (B5), Eq. (B3) in terms of the new variable takes the form of the hypergeometric differential equation,

$$
z(1-z) \frac{\partial^{2} w}{\partial z^{2}}+[c-(a+b+1) z] \frac{\partial w}{\partial z}-a b w=0
$$

with the parameters

$$
\begin{gathered}
a=\frac{1}{2 \gamma}\left((l+2) \gamma-1+\sqrt{1+\gamma^{2} l^{2}+2 \gamma \frac{\omega^{2}}{\omega_{0}^{2}}}\right), \\
b=\frac{1}{2 \gamma}\left((l+2) \gamma-1-\sqrt{1+\gamma^{2} l^{2}+2 \gamma \frac{\omega^{2}}{\omega_{0}^{2}}}\right), \\
c=l+1 .
\end{gathered}
$$

The solution of this equation for each component of the trapped Fermi gas leads to the expression for the distribution of the density oscillations,

$$
\begin{aligned}
\delta n_{j}(r, l, \omega)= & A_{j} r^{l} F\left(a_{j}, b_{j} ; l+1 ; \frac{r^{2}}{R_{j}^{2}}\right) \\
& +B_{j} r^{l} F\left(a_{j}, b_{j} ; 2-\frac{1}{\gamma_{j}} ; 1-\frac{r^{2}}{R_{j}^{2}}\right) .
\end{aligned}
$$

The number of independent coefficients $A_{j}, B_{j}$ can be reduced using the boundary conditions at $r=0$ and at $r=$ $R_{p}(p=\uparrow, \downarrow)$. The density oscillation amplitude for the quasicondensate must be finite at $r=0$, so that $B_{S}=0$. When the equilibrium density of the normal phase vanishes, also the oscillations of the density must turn to zero. Hence the density oscillations for the normal phase must tend to zero at the outer boundary $R_{p}$ for each normal component. This leads to the condition

$$
A_{p} F\left(a_{p}, b_{p} ; l+1 ; 1\right)+B_{p}=0 \text { for } \quad p=\uparrow, \downarrow .
$$

After some algebra with hypergeometric functions, we arrive at the following solutions for the superfluid and normal phases:

$$
\begin{aligned}
\delta n_{S}(r, l, \omega) & =A_{S} r^{l} F\left(a_{S}, b_{S} ; l+1 ; \frac{r^{2}}{R_{S}^{2}}\right), \\
\delta n_{p}(r, l, \omega)= & A_{p} r^{l}\left(1-\frac{r^{2}}{R_{p}^{2}}\right)^{\frac{1}{\gamma_{p}}-1} \\
& \times F\left(l+1-a_{p}, l+1-b_{p} ; \frac{1}{\gamma_{p}} ; 1-\frac{r^{2}}{R_{p}^{2}}\right) .
\end{aligned}
$$

In the zero-temperature limit, these expressions explicitly tend to the corresponding formulas obtained in Ref. [23].

\section{APPENDIX C: SET OF EQUATIONS FOR COLLECTIVE MODES}

Here, the set of equations following from the boundary conditions is derived. First, let us consider the equation based on the boundary condition for the pressure (35). As follows from Eqs. (27) and (28) jointly with the polytropic equation of state (10), the vibrational amplitudes for the pressure and for the chemical potential of the $j$ th component $\delta P_{j}(r, l, \omega)$ are proportional to the vibrational amplitude for the density:

$$
\begin{gathered}
\delta P_{j}(r, l, \omega)=\left(\gamma_{j}+1\right) C_{j}\left[n_{j}(r)\right]^{\gamma_{j}} \delta n_{j}(r, l, \omega), \\
\delta \mu_{j}(r, l, \omega)=\left(\gamma_{j}+1\right) C_{j}\left[n_{j}(r)\right]^{\gamma_{j}-1} \delta n_{j}(r, l, \omega),
\end{gathered}
$$

where $n_{j}(r)$ is the equilibrium distribution of the density of the $j$ th component. The boundary condition (35) then results in the following equation, involving the amplitudes of the density oscillations at the boundary $\delta n_{j}\left(R_{b}, l, \omega\right)$ and the mechanical displacement of the core boundary $\delta R(l, \omega)$ :

$$
\begin{aligned}
& \left(\gamma_{S}+1\right) C_{S}\left[n_{S}\left(R_{b}\right)\right]^{\gamma_{S}} \delta n_{S}\left(R_{b}, l, \omega\right) \\
& \quad-\left(\gamma_{\uparrow}+1\right) C_{\uparrow}\left[n_{\uparrow}\left(R_{b}\right)\right]^{\gamma_{\uparrow}} \delta n_{\uparrow}\left(R_{b}, l, \omega\right) \\
& \quad-\left(\gamma_{\downarrow}+1\right) C_{\downarrow}\left[n_{\downarrow}\left(R_{b}\right)\right]^{\gamma_{\downarrow}} \delta n_{\downarrow}\left(R_{b}, l, \omega\right) \\
& \quad+\left.\left(\frac{\partial P_{S}(r)}{\partial r}-\frac{\partial P_{\uparrow}(r)}{\partial r}-\frac{\partial P_{\downarrow}(r)}{\partial r}\right)\right|_{r=R_{b}} \delta R(l, \omega)=0 .
\end{aligned}
$$

Next, we rewrite the boundary condition for the chemical potential (36) by substituting (C2):

$$
\begin{aligned}
& 2\left(\gamma_{S}+1\right) C_{S}\left[n_{S}\left(R_{b}\right)\right]^{\gamma_{S}-1} \delta n_{S}\left(R_{b}, l, \omega\right) \\
& \quad-\left(\gamma_{\uparrow}+1\right) C_{\uparrow}\left[n_{\uparrow}\left(R_{b}\right)\right]^{\gamma_{\uparrow}-1} \delta n_{\uparrow}\left(R_{b}, l, \omega\right) \\
& \quad-\left(\gamma_{\downarrow}+1\right) C_{\downarrow}\left[n_{\downarrow}\left(R_{b}\right)\right]^{\gamma_{\downarrow}-1} \delta n_{\downarrow}\left(R_{b}, l, \omega\right)=0 .
\end{aligned}
$$


In the same way, two equations are obtained based on the boundary conditions (37) for the current flow:

$$
\begin{gathered}
\left.2 n_{p}\left(R_{b}\right) \frac{\partial\left[\delta \mu_{p}(r, l, \omega)\right]}{\partial r}\right|_{r=R_{b}}-\left.n_{S}\left(R_{b}\right) \frac{\partial\left[\delta \mu_{S}(r, l, \omega)\right]}{\partial r}\right|_{r=R_{b}} \\
=m \omega^{2}\left[2 n_{p}\left(R_{b}\right)-n_{S}\left(R_{b}\right)\right] \delta R(l, \omega) \text { for } \quad p=\uparrow, \downarrow .
\end{gathered}
$$

Therefore, we arrive at the uniform set of four linear equations for four variables $A_{S}, A_{\uparrow}, A_{\downarrow}, \delta R$. The eigenfrequencies are determined as the roots of the secular equation

$$
\operatorname{det}\left\|f_{j, k}(\omega)\right\|=0,
$$

where the matrix elements $f_{j, k}(\omega)$ of the dynamic matrix for collective modes are obtained immediately from Eqs. (C3)(C5) when substituting there the solution (31) and (32).
[1] M. Greiner, O. Mandel, T. Esslinger, T. W. Hänsch, and I. Bloch, Nature (London) 415, 39 (2002).

[2] M. Greiner, O. Mandel, T. W. Hänsch, and I. Bloch, Nature (London) 419, 51 (2002).

[3] J. K. Chin, D. E. Miller, Y. Liu, C. Stan, W. Setiawan, C. Sanner, K. Xu, and W. Ketterle, Nature (London) 443, 961 (2006).

[4] V. L. Berezinskii, Sov. Phys. JETP 32, 493 (1971).

[5] J. M. Kosterlitz and D. J. Thouless, J. Phys. C 6, 1181 (1973); J. M. Kosterlitz, ibid. 7, 1046 (1974).

[6] D. R. Nelson and J. M. Kosterlitz, Phys. Rev. Lett. 39, 1201 (1977).

[7] Z. Hadzibabic, P. Krüger, M. Cheneau, B. Battelier, and J. Dalibard, Nature (London) 441, 1118 (2006).

[8] P. Krüger, Z. Hadzibabic, and J. Dalibard, Phys. Rev. Lett. 99, 040402 (2007).

[9] I. Bloch, J. Dalibard, and W. Zwerger, Rev. Mod. Phys. 80, 885 (2008).

[10] S. S. Botelho and C. A. R. Sá de Melo, Phys. Rev. Lett. 96, 040404 (2006).

[11] W. Zhang, G.-D. Lin, and L.-M. Duan, Phys. Rev. A 78, 043617 (2008).

[12] E. Babaev and H. Kleinert, Phys. Rev. B 59, 12083 (1999).

[13] J. Tempere, S. N. Klimin, and J. T. Devreese, Phys. Rev. A 79, 053637 (2009).

[14] J. Tempere, S. N. Klimin, and J. T. Devreese, Physica C 470, 809 (2010).

[15] J. Kinast, S. L. Hemmer, M. E. Gehm, A. Turlapov, and J. E. Thomas, Phys. Rev. Lett. 92, 150402 (2004).

[16] J. Kinast, A. Turlapov, and J. E. Thomas, Phys. Rev. A 70, 051401(R) (2004).

[17] M. Bartenstein, A. Altmeyer, S. Riedl, S. Jochim, C. Chin, J. H. Denschlag, and R. Grimm, Phys. Rev. Lett. 92, 203201 (2004).

[18] A. Altmeyer, S. Riedl, M. J. Wright, C. Kohstall, J. H. Denschlag, and R. Grimm, Phys. Rev. A 76, 033610 (2007).

[19] A. Altmeyer, S. Riedl, C. Kohstall, M. J. Wright, R. Geursen, M. Bartenstein, C. Chin, J. H. Denschlag, and R. Grimm, Phys. Rev. Lett. 98, 040401 (2007).

[20] M. J. Wright, S. Riedl, A. Altmeyer, C. Kohstall, E. R. Sánchez Guajardo, J. H. Denschlag, and R. Grimm, Phys. Rev. Lett. 99, 150403 (2007).

[21] S. Riedl, E. R. Sanchez Guajardo, C. Kohstall, A. Altmeyer, M. J. Wright, J. H. Denschlag, R. Grimm, G. M. Bruun, and H. Smith, Phys. Rev. A 78, 053609 (2008).

[22] A. Lazarides and B. Van Schaeybroeck, Phys. Rev. A 77, 041602(R) (2008).

[23] B. Van Schaeybroeck, J. Tempere, and A. Lazarides, e-print arXiv:0911.0984.

[24] H. Heiselberg, Phys. Rev. Lett. 93, 040402 (2004).
[25] N. Manini and L. Salasnich, Phys. Rev. A 71, 033625 (2005).

[26] A. Bulgac and G. F. Bertsch, Phys. Rev. Lett. 94, 070401 (2005).

[27] G. E. Astrakharchik, R. Combescot, X. Leyronas, and S. Stringari, Phys. Rev. Lett. 95, 030404 (2005).

[28] J. Yin, Y. L. Ma, and G. Huang, Phys. Rev. A 74, 013609 (2006).

[29] Y. Zhou and G. Huang, Phys. Rev. A 75, 023611 (2007).

[30] W. Wen and G. Huang, Phys. Lett. A 362, 331 (2007).

[31] M. Cozzini and S. Stringari, Phys. Rev. Lett. 91, 070401 (2003).

[32] H. Hu, A. Minguzzi, X. J. Liu, and M. P. Tosi, Phys. Rev. Lett. 93, 190403 (2004).

[33] P. Nozières and S. Schmitt-Rink, J. Low Temp. Phys. 59, 195 (1985).

[34] C. A. R. Sá de Melo, M. Randeria, and J. R. Engelbrecht, Phys. Rev. Lett. 71, 3202 (1993).

[35] A. Perali, P. Pieri, L. Pisani, and G. C. Strinati, Phys. Rev. Lett. 92, 220404 (2004).

[36] R. B. Diener, R. Sensarma, and M. Randeria, Phys. Rev. A 77, 023626 (2008).

[37] J. Tempere, S. N. Klimin, J. T. Devreese, and V. V. Moshchalkov, Phys. Rev. B 77, 134502 (2008).

[38] J. Tempere, S. N. Klimin, and J. T. Devreese, Phys. Rev. A 78, 023626 (2008)

[39] S. Schmitt-Rink, C. M. Varma, and A. E. Ruckenstein, Phys. Rev. Lett. 63, 445 (1989).

[40] P. C. Hohenberg, Phys. Rev. 158, 383 (1967).

[41] N. D. Mermin and H. Wagner, Phys. Rev. Lett. 17, 1133 (1966).

[42] Yu. Kagan, B. V. Svistunov and G. V. Shlyapnikov, Zh. Eksp. Teor. Fiz. 93, 552 (1987) [Sov. Phys. JETP 66, 314 (1987)].

[43] J. Tempere, M. Wouters, and J. T. Devreese, Phys. Rev. B 75, 184526 (2007).

[44] M. Randeria, J. M. Duan, and L. Y. Shieh, Phys. Rev. B 41, 327 (1990).

[45] A. I. Safonov, S. A. Vasilyev, I. S. Yasnikov, I. I. Lukashevich, and S. Jaakkola, Phys. Rev. Lett. 81, 4545 (1998).

[46] L.-K. Lim, C. M. Smith, and H. T. C. Stoof, Phys. Rev. A 78, 013634 (2008).

[47] C. J. Pethick and H. Smith, Bose-Einstein Condensation in Dilute Gases (Cambridge University Press, Cambridge, 2002).

[48] S. Giorgini, L. P. Pitaevskii, and S. Stringari, Rev. Mod. Phys. 80, 1215 (2008).

[49] L. D. Landau and L. M. Lifshitz, Course of Theoretical Physics (Butterworth-Heinemann, London, 1987), Vols. VI and IX.

[50] M. Cozzini and S. Stringari, Phys. Rev. Lett. 91, 070401 (2003).

[51] J. F. Dobson, Phys. Rev. Lett. 73, 2244 (1994).

[52] E. Taylor, A. Griffin, and Y. Ohashi, Phys. Rev. A 76, 023614 (2007). 